\title{
RGMB-AS1/miR-22-3p/NFIB axis contributes to the progression of gastric cancer
}

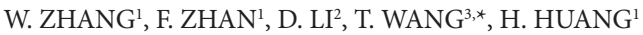 \\ ${ }^{1} 2^{\text {nd }}$ Department of General Surgery, Wuwei People's Hospital, Wuwei, Gansu, China; ${ }^{2}$ Clinical Laboratory, Wuwei People's Hospital, Wuwei, \\ Gansu, China; ${ }^{3}$ Head and Neck Surgery Department, Gansu Provincial Cancer Hospital, Lanzhou, Gansu, China
}

*Correspondence: 2208656401@qq.com

Received April 18, 2019 / Accepted July 21, 2019

\begin{abstract}
Gastric cancer (GC), one of the most common cancers worldwide, presents a considerable threat to human health. Although multiple investigations have been made to figure out therapies for this disease, the prognosis of patients suffered from GC remains poor. It has been reported that lncRNAs exerted their significant effects on numerous cancers. Evidence confirmed that lncRNA RGMB-AS1 played an oncogenic role in the progression of cancers. However, the biological function and molecular mechanism of RGMB-AS1 in GC haven't been explored. In this study, our results demonstrated that RGMB-AS1 was upregulated in GC cells and knockdown of RGMB-AS1 suppressed cell proliferation, migration, invasion, EMT process and promoted cell apoptosis. Molecular mechanism experiments indicated that RGMB-AS1 could bind with miR-22-3p and NFIB was a downstream target gene of miR-22-3p. Additionally, RGMB-AS1 suppression upregulated the expression of miR-22-3p and miR-22-3p inhibitor could reverse the inhibitive role of sh-RGMB-AS1-1 in NFIB expression. Rescues assays showed that NFIB overexpression partially recovered the inhibitory function on cell proliferation, migration, invasion, EMT process and the promotive function on cell apoptosis caused by RGMB-AS1 depletion. Taken together, RGMB-AS1 contributes to the progression of GC by regulating miR-22-3p/NFIB axis, indicating a new therapeutic target for $\mathrm{GC}$ treatment.
\end{abstract}

Key words: RGMB-AS1, miR-22-3p, NFIB, gastric cancer

Gastric cancer (GC) is deemed as one of the main causes of cancer-related mortality worldwide, which shows the second highest incidence in males. GC accounts for $8 \%$ of all cancer deaths [1]. Both genetic and environmental factors contribute to the occurrence of GC. Researches have shown that several key lncRNAs and miRNAs are involved in modulating drug resistance and can serve as potential biomarkers for predicting drug resistance and prognosis in GC $[2,3]$. Salty diet, lack of intake of fresh vegetables and fruit, smoking, alcoholic beverage, and other factors serve as important environmental stimuli of GC [4]. Various biomarkers of GC have been studied, while the underlying molecular regulation mechanism of the particular biomarkers has not been fully explored. Therefore, it is of great consequence to delve into the underlying molecular regulation mechanism of the specific biomarkers in the development of GC.

Long noncoding RNA (lncRNA) is distinguished from other RNAs by its length of more than 200 nucleotides and no protein-coding ability $[5,6]$. Increasing evidence confirms that lncRNAs play significant roles in the tumorigenesis and development of GC. For instance, lncRNA OR3A4 exerts its oncogenic function by promoting tumor progression in GC [7]. LncRNA XIST aggravates GC progression by competitively sponging miR-101 and targeting EZH2 [8]. Increasing evidence indicates that RGMB-AS1 functions as a tumorpromotor in various cancers. For instance, RGMB-AS1 contributes to the progression of lung adenocarcinoma by regulating RGMB expression through exon2 of RGMB [9]. LncRNA RGMB-AS1 expression was significantly higher in NSCLC tissues than in adjacent non-tumor tissues [10]. Although the biological role of RGMB-AS1 in various cancers has been explored, the molecular mechanism and function of it in GC remain obscure.

In this study, we planned to research whether lncRNA RGMB-AS1 exhibited an oncogenic role in GC. Finally, we confirmed that RGMB-AS1 contributed to the progression of GC through miR-22-3p/NFIB axis. 


\section{Materials and methods}

Cell culture and transfection. GC cell lines (AGS, BGC-823, SGC7901, MGC-803) and human immortalized gastric epithelial cell line (GES-1) were purchased from the American Type Culture Collection (ATCC; Manassas, VA, USA). All cells were cultured in Roswell Park Memorial Institute (RPMI) 1640 medium added with $10 \%$ fetal bovine serum (FBS; Gibco/Invitrogen Inc., Carlsbad, CA, USA), streptomycin $(100 \mathrm{mg} / \mathrm{ml})$, and penicillin $(100 \mathrm{U} / \mathrm{ml})$, and then they were cultured at $37^{\circ} \mathrm{C}$ in humidified atmosphere with $5 \% \mathrm{CO}_{2}$.

In order to downregulate the expression of RGMB-AS1 in cells, short hairpin RNAs (shRNAs) explicitly targeting RGMB-AS1 were designed and synthesized by GenePharma (Shanghai, China). The full-length sequences of RGMB-AS1 and NFIB were correspondingly synthesized and subcloned into pcDNA3.1 vectors (Invitrogen, Carlsbad, USA) to yield pcDNA3.1/RGMB-AS1 and pcDNA3.1/NFIB. MiR-22-3p mimics, miR-22-3p inhibitor, and the corresponding negative controls (NC mimics and $\mathrm{NC}$ inhibitor) were acquired from GenePharma. All plasmids were transfected into AGS and BGC-823 cells using Lipofectamine 2000 (Invitrogen, Carlsbad, CA, USA) following the instructions of the manufacturer.

RNA extraction and quantitative real-time PCR. Employing Trizol reagent (Takara, Otsu, Japan), total RNA was extracted from AGS or BGC-823 cells. RNAs were inversely transcribed into cDNA by utilizing $\mathrm{TaqMan}^{\mathrm{TM}}$ Advanced miRNA cDNA Synthesis Kit (Waltham, MA, USA) or the reverse transcription kit (Takara, Otsu, Japan). The RT-qPCR was carried out by SYBR Green PCR Kit (Takara, Otsu, Japan). Internal controls were GAPDH and U6. The outcomes of RT-qPCR were evaluated by utilizing Applied Biosystems Step One Plus Real-Time PCR System (Applied Biosystems, Foster City, USA), and these relative expression levels were examined by $2^{-\Delta \Delta \mathrm{Ct}}$ method.

CCK-8 assay. Cell-Counting Kit 8 (CCK8; Dojindo Molecular Technologies) was applied to gauge the proliferation ability of GC cells. The transfected cells $\left(1 \times 10^{3}\right.$ cells/ well) were seeded in 96 -well plates. CCK-8 solution $(10 \mu \mathrm{l})$ was added to each well after an incubation of $0,24,48,72$ and $96 \mathrm{~h}$, followed by an incubation lasting $4 \mathrm{~h}$. The absorbance at $450 \mathrm{~nm}$ was identified by utilizing a Multiskan Go spectrophotometer (Thermo Fisher Scientific, Inc.).

Flow cytometry analysis. In brief, transfected cells were collected and resuspended with phosphate-buffered saline (PBS). Afterward, the density of $1 \times 10^{6} \mathrm{cells} / \mathrm{ml}$ was prepared. In line with the manufacturer's instruction, transfected cells were double-stained by propidium iodide and Annexin V-FITC. Finally, cell apoptosis was identified utilizing flow cytometry (BD Biosciences, Franklin Lakes, NJ).

Transwell assay. Transwell assay was utilized to examine cell migration. Transwell chambers (Corning Incorporated, Corning, NY, USA) without (for migration assay) or with (for invasion assay) matrigel (BD Biosciences, Bedford, MA, USA) were utilized for transwell assay. The upper chamber was filled with $200 \mu \mathrm{l}$ RPMI 1640 medium containing transfected cells $\left(1 \times 10^{3}\right.$ cells/well $)$ while the lower chamber was filled with $800 \mu \mathrm{l}$ RPMI 1640 medium containing 10\% FBS. After $48 \mathrm{~h}$ of incubation, migrated or invaded cells were fixed by methanol and stained utilizing $0.5 \%$ crystal violet (Amresco Co., Solon, OH, USA). Then, stained cells were counted under a light microscope (Olympus Corporation, Tokyo, Japan).

RNA immunoprecipitation. RIP assay was carried out by using Magna RNA-binding protein immunoprecipitation kit (Millipore, Billerica, MA, USA). Cell lysate (AGS and BGC-823) was incubated in RIP buffer containing magnetic beads which were conjugated with Ago2 antibodies. IgG was utilized as a control. Proteinase $\mathrm{K}$ was utilized to isolate immunoprecipitated RNAs. Then, purified RNAs were identified by RT-qPCR.

Luciferase reporter assay. The 3'-UTR sequences of NFIB containing the predicted miR-22-3p binding sites and full-length sequences of RGMB-AS1 were subcloned into the pmirGLO vector (Promega, Madison, WI, USA) to produce the wild-type NFIB reporter (pmirGLO-NFIB$\mathrm{Wt}$ ) and the wild-type RGMB-AS1 reporter (pmirGLORGMB-AS1-Wt). The mutant-type RGMB-AS1 reporter (pmirGLO-RGMB-AS1-Mut) and the mutant-type NFIB reporter (pmirGLO-NFIB-Mut) were provided by GeneArt ${ }^{\mathrm{Tm}}$ Site-Directed Mutagenesis System (Thermo Fisher Scientific). MiR-22-3p mimics or NC mimics was co-transfected with RGMB-AS1-Wt or RGMB-AS1-Mut into AGS or BGC-823 cells. NC mimics, miR-22-3p mimics or miR-22-3p mimics+pcDNA3.1/RGMB-AS1 were also co-transfected with NFIB-Wt or NFIB-Mut into AGS or BGC-823 cells. After an incubation lasting 48 hours, the luciferase activities were evaluated by the application of the luciferase reporter assay system (Promega, Madison WI, USA).

Western blot. Proteins were extracted using RIPA lysis buffer (Beyotime Biotechnology, China) with protease inhibitors (Roche, China). Then the concentration of these proteins was determined with the employment of $\mathrm{BCA}^{\mathrm{m}}$ Protein Assay Kit (Pierce, Appleton, USA). Proteins were separated by sodium dodecyl sulfate-polyacrylamide gel electrophoresis (SDS-PAGE), and then transferred onto the polyvinylidene difluoride (PVDF) membranes. After blocking by skim milk, the primary antibodies were employed to incubate membranes overnight at $4{ }^{\circ} \mathrm{C}$. The primary antibodies were all purchased from Abcam Company (Abcam, Cambridge, UK) as follows: E-cadherin (ab194982), N-cadherin (ab18203), Vimentin (ab92547), GAPDH (ab8265), and NFIB (ab186738). After that, these membranes were incubated with secondary antibody for $2 \mathrm{~h}$ at room temperature. The signals were captured via the utilization of the chemiluminescent detection system. Internal control was GADPH.

Ethynyl deoxyuridine (EdU) incorporation assay. Cell proliferation was assessed via employing EdU kit (C10310, 
Ribobio, Guangzhou, China). Cells were seeded into 96-well plates, and $100 \mu \mathrm{l}$ of EdU reagent was supplemented to each well for $2 \mathrm{~h}$ of incubation. After washing with PBS, the cells were fixed in $50 \mu \mathrm{l}$ of fixation buffer that contains about 4\% paraformaldehyde, decolored with $2 \mathrm{mg} / \mathrm{ml}$ glycine, and permeated with $100 \mu \mathrm{l}$ of penetrant for decolorization shaker incubation. After washing with PBS once, the cells were stained with $100 \mu$ of Apollo in the dark for 30 min to determine the specific reaction between Apollo dye and EdU. Then, the cells were washed with penetrant and methanol. Subsequently, the cells were incubated with $100 \mu \mathrm{l}$ of $1 \times$ Hoechst 33342 , followed by viability determination under a fluorescent microscope.
Fluorescence in situ hybridization assays. AGS and BGC-823 cells were fixed in $4 \%$ formaldehyde for $15 \mathrm{~min}$ and then washed with PBS. Pepsin ( $1 \%$ in $10 \mathrm{mM} \mathrm{HCl}$ ) was used to treat the fixed cells, followed by continuous dehydration with ethanol. The dried cells were mixed with $40 \mathrm{nM}$ of the FISH probe (Hoechst, RGMB-AS1, miR-22-3p) in a hybridization buffer and incubated at $80^{\circ} \mathrm{C}$ for $2 \mathrm{~min}$. After being left to stand at $55^{\circ} \mathrm{C}$ for $2 \mathrm{~h}$, the slides were washed and dehydrated, and finally observed and detected with Prolong Gold Antifade Reagent using Hoechst. The RNA FISH probe was supplied by Ribobio. The probe sequences were as listed: miR-22-3p: CUCACCUGAACUCUGCCGUU;
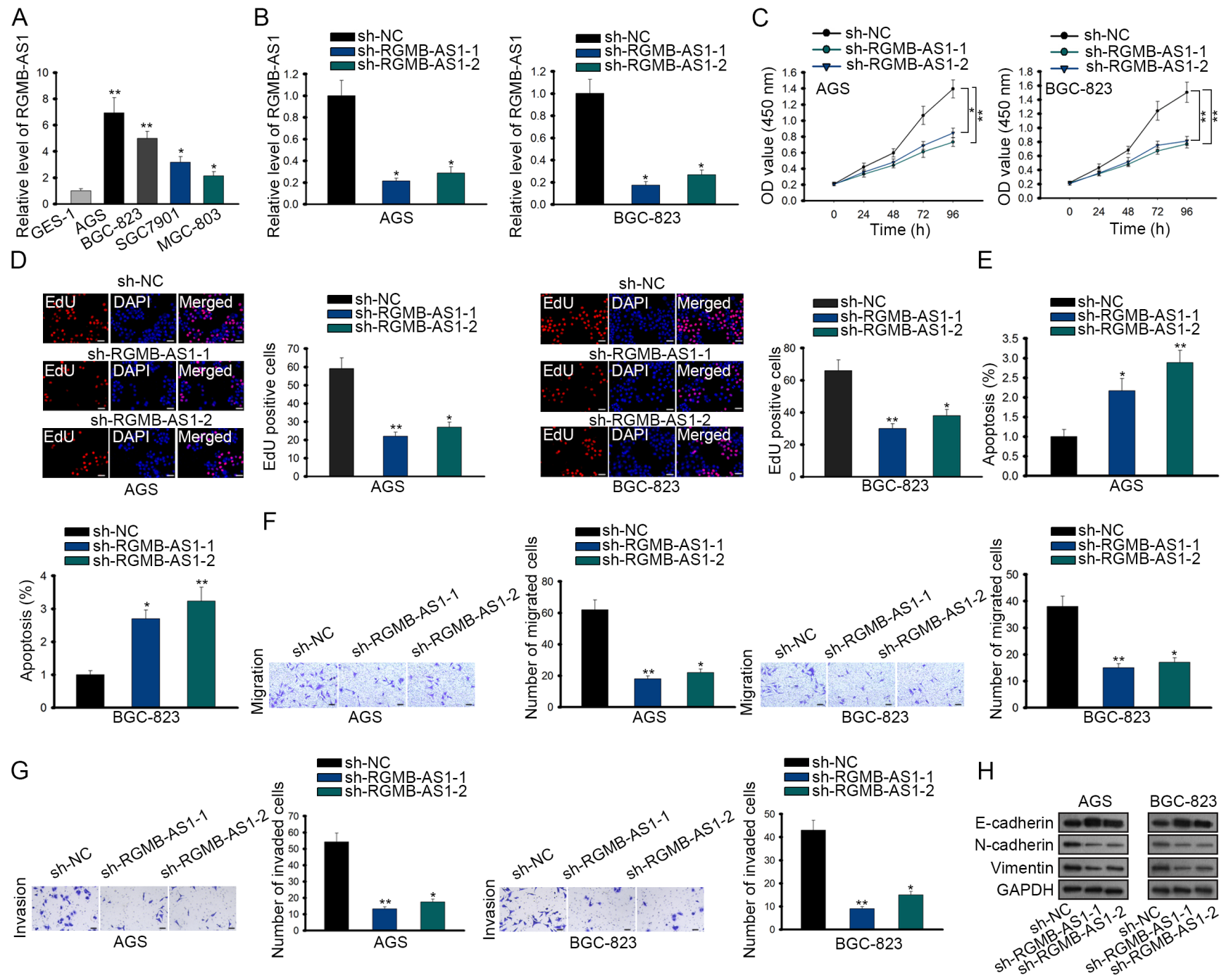

Figure 1. RGMB-AS1 expression is upregulated in GC cell lines and RGMB-AS1 knockdown inhibits the progression of GC. A) RT-qPCR was conducted to detect the expression of RGMB-AS1 in normal gastric cells and GC cells. B) The knockdown efficiency of sh-RGMB-AS1-1 and sh-RGMBAS1-2 was analyzed through RT-qPCR. C and D) Cell proliferation in GC cells was determined by CCK-8 and EdU assays (scale bar $=20 \mu \mathrm{m})$. E) Flow cytometry analysis was performed to examine cell apoptosis in GC cells. F and G) Transwell assay was carried out to evaluate cell migration and invasion in GC cells (scale bar $=20 \mu \mathrm{m}) . \mathrm{H}$ ) Western blot assay was performed to detect the expression of EMT process related proteins $(\mathrm{E}$-cadherin, $\mathrm{N}$-cadherin and Vimentin). ${ }^{*} \mathrm{p}<0.05,{ }^{\star *} \mathrm{p}<0.01$. 
A

$\begin{array}{cc}\text { RGMB-ASL-WT } & \text { 5'-ggAUUAAUCAA-UGGCAGCUc-3' } \\ & |||||||||||| \\ \text { Hsa-miR-22-3p } & \text { 3'-ugUCAAGAAGUUGACCGUCGAa-5' } \\ \text { RGMB-AS1-Mut } & \text { 5'-ggAUUAAAAGUU-ACCGUCGAc-3' }\end{array}$

B

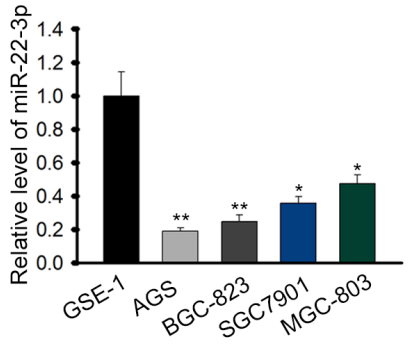

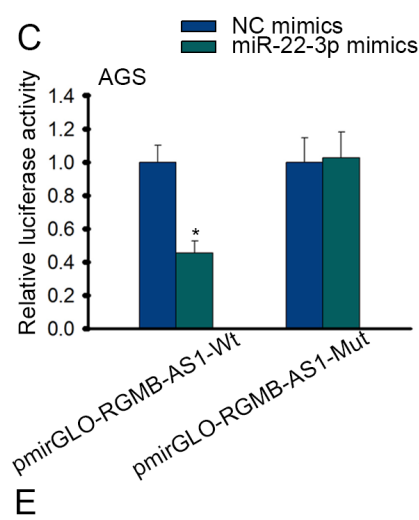
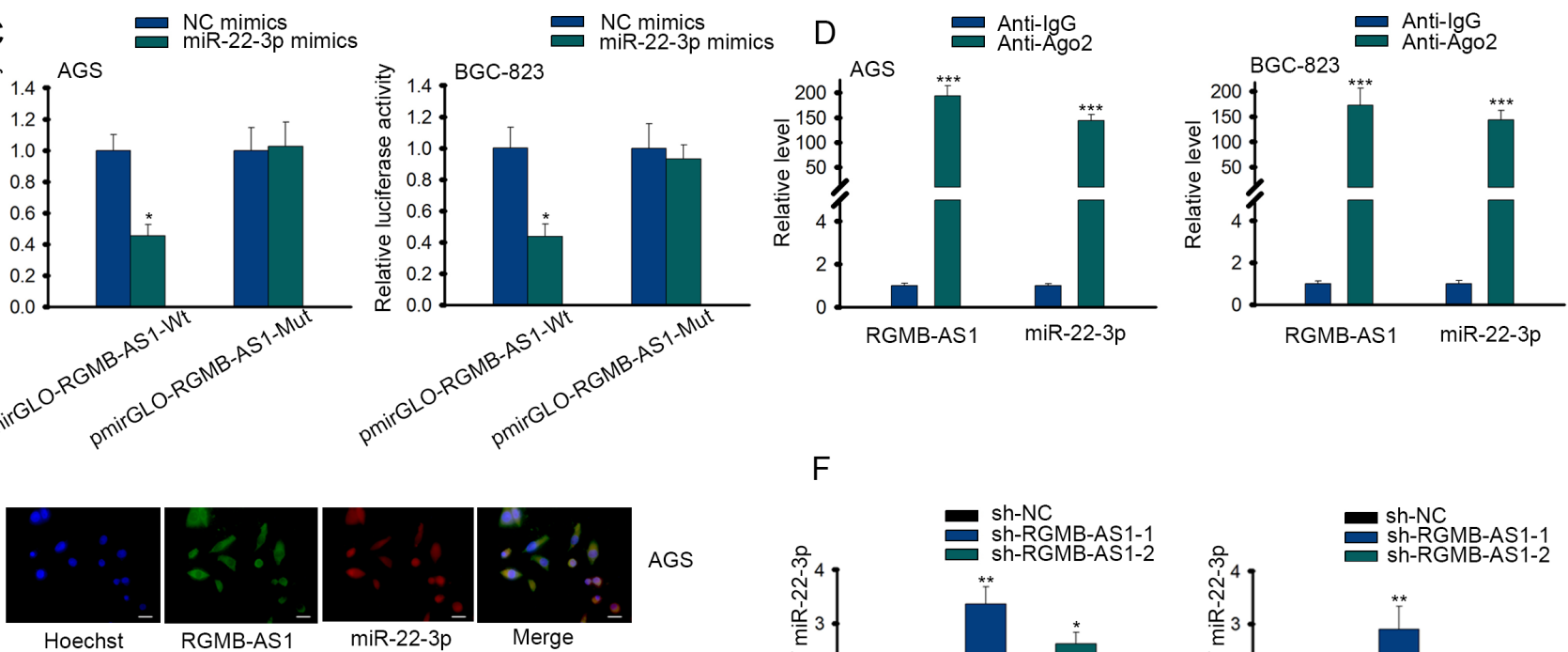

Hoechst

RGMB-AS1

miR-22-3p

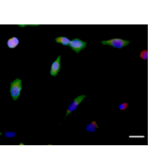

BGC-823
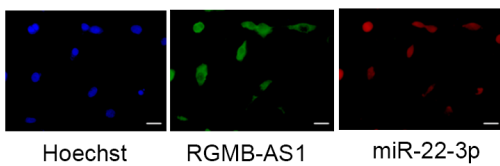

miR-22-3p

Merge
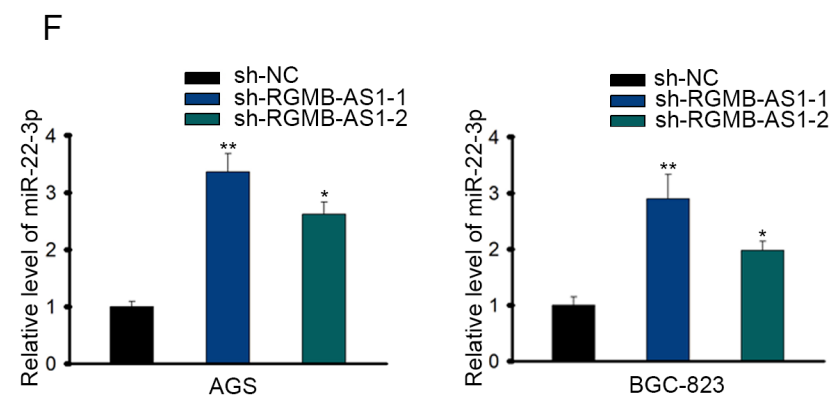

Figure 2. RGMB-AS1 serves as a sponge of miR-22-3p in GC. A) The potential binding sites between miR-22-3p and RGMB-AS1 were predicted by starBase v2.0 software. B) MiR-22-3p expression in normal gastric cells and GC cells was investigated by RT-qPCR. C and D) Using luciferase reporter and RIP assays, RGMB-AS1 was confirmed to bind with miR-22-3p. E) RNA-FISH analysis identified the location of RGMB-AS1 and miR-22-3p in cells $($ scale bar $=20 \mu \mathrm{m})$. F) RT-qPCR was utilized for assessing the expression of RGMB-AS1 and miR-22-3p in GC cells. ${ }^{\star} \mathrm{p}<0.05,{ }^{\star *} \mathrm{p}<0.01,{ }^{\star * *} \mathrm{p}<0.001$.

Statistical analysis. All data were shown as mean \pm standard deviation (SD). SPSS 22.0 software (SPSS, Chicago, IL, USA) was used for statistical analysis. Each experiment was performed 3 times. Differences among groups were determined by the use of student's t-test or one-way ANOVA analysis. The level of significance $\mathrm{p}<0.05$ was considered statistically significant.

\section{Results}

RGMB-AS1 expression is upregulated in GC cells and RGMB-AS1 suppression inhibits the progression of GC. To assess the expression of RGMB-AS1 in GC cells, RT-qPCR was performed, indicating that the RGMB-AS1 expression was apparently upregulated in GC cells compared with normal gastric cells (Figure 1A). Knockdown of RGMB-AS1 by using sh-RGBM-AS1-1 or sh-RGBM-AS1-2 caused a remarkable decline of the RGMB-AS1 expression in GC cells (Figure 1B). Additionally, CCK-8 assay displayed that the cell proliferation was declined by RGMB-AS1 knockdown in AGS and BGC-823 cells (Figure 1C). EdU assay showed that the RGMB-AS1 knockdown suppressed cell proliferation in GC (Figure 1D). Furtherly, flow cytometry analysis was utilized to detect cell apoptosis in GC cells, presenting that knockdown RGMB-AS1 notably increased cell apoptosis (Figure 1E). As shown in the transwell assay, the capability of cell migration or invasion was obviously reduced by the RGMB-AS1 silence (Figures $1 F-G$ ). Results from western blot assay exhibited that the E-cadherin expression was enhanced, $\mathrm{N}$-cadherin and Vimentin expression were reduced by the RGMB-AS1 suppression (Figure $1 \mathrm{H}$ ). In conclusion, the RGMB-AS1 expression is upregulated in GC cells and the RGMB-AS1 suppression inhibits the progression of GC cells. 


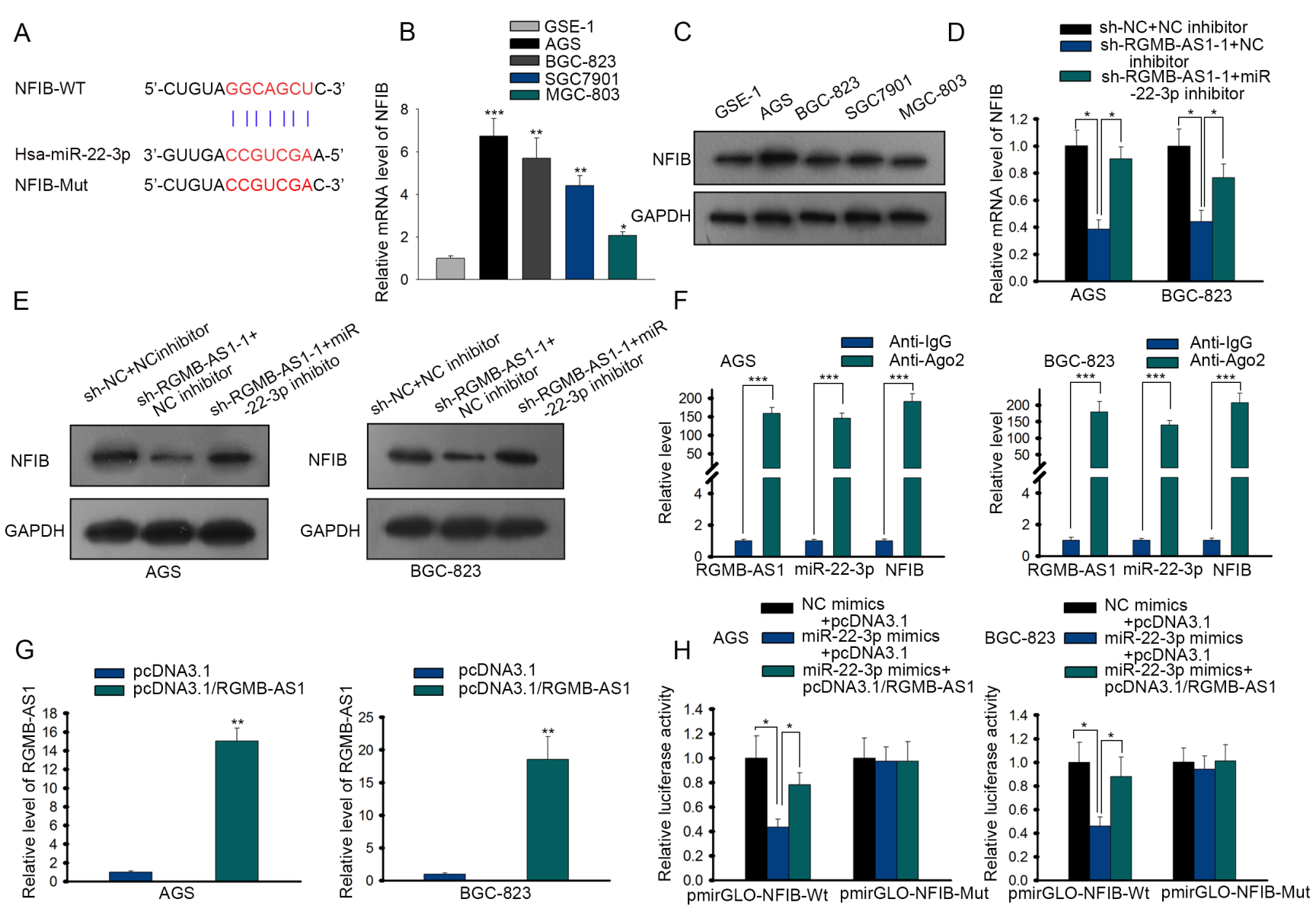

Figure 3. RGMB-AS1 modulates miR-22-3p to regulate NFIB in GC. A) The potential binding sites between miR-22-3p and NFIB were hypothesized through starBase v2.0 software. B and C) RT-qPCR and western blot assays were performed to check the expression of NCIB in normal gastric cells and GC cells. D and E) RT-qPCR and western blot assays were performed to uncover the interaction among RGMB-AS1, miR-22-3p and NFIB. F) RIP assay was used to unveil RGMB-AS1 modulated NFIB expression by sponging miR-22-3p. G) The expression of RGMB-AS1 was tested after the RGMB-AS1 overexpression through RT-qPCR. H) Luciferase reporter analysis was employed to further verify RGMB-AS1 modulated NFIB expression by sponging miR-22-3p. ${ }^{*} p<0.05,{ }^{* *} p<0.01,{ }^{* * *} p<0.001$.

RGMB-AS1 can combine with miR-22-3p in GC. We further investigated the underlying mechanism of RGBM-AS1 in GC cells. Through searching starBase, miR-22-3p was found to have a binding site for RGBM-AS1 (Figure 2A). Then the expression of miR-22-3p in GC cells and normal gastric cells was checked by RT-qPCR, disclosing that the miR-22-3p expression was lower in GC cells (Figure 2B). In addition, the luciferase reporter analysis presented that the luciferase activity of RGMB-AS1-WT was notably declined by miR-22-3p mimics compared with control, while RGMB-AS1-Mut disclosed no distinct change between the miR-22-3p mimics group and the NC mimics group (Figure 2C). Furthermore, RIP assay showed that both RGMB-AS1 and miR-22-3p were enriched in Ago2-conjugated beads (Figure 2D). According to the FISH assay, RGMB-AS1 and miR-22-3p were discovered to be both located in the cytoplasm (Figure 2E). Finally, with the employment of RT-qPCR, suppression of RGMB-AS1 promi- nently increased the expression of miR-22-3p (Figure $2 \mathrm{~F}$ ). All the results revealed that RGMB-AS1 can combine with miR-22-3p in GC.

RGMB-AS1 sponges miR-22-3p to regulate NFIB expression in GC. LncRNAs were reported to act as a ceRNA to bind with miRNAs to regulating mRNAs expression in several cancers, so we investigated whether RGMB-AS1 also had this function. According to starBase, NFIB was found to have a binding site for miR-22-3p (Figure 3A). Then RT-qPCR and WB assays were employed to determine the expression of NFIB in GC cells, exhibiting that mRNA and protein expression of NFIB were extremely higher in GC cells (Figures 3B-C). Furtherly, as shown in Figures 3D and 3E, the knockdown of RGMB-AS1 repressed the mRNA and protein expression of NFIB while the miR-22-3p inhibitor could restore the inhibitory effect caused by RGMB-AS1 knockdown. Moreover, RGMB-AS1, miR-22-3p, and NFIB were immunoprecipitated by anti-Ago2 but not the 

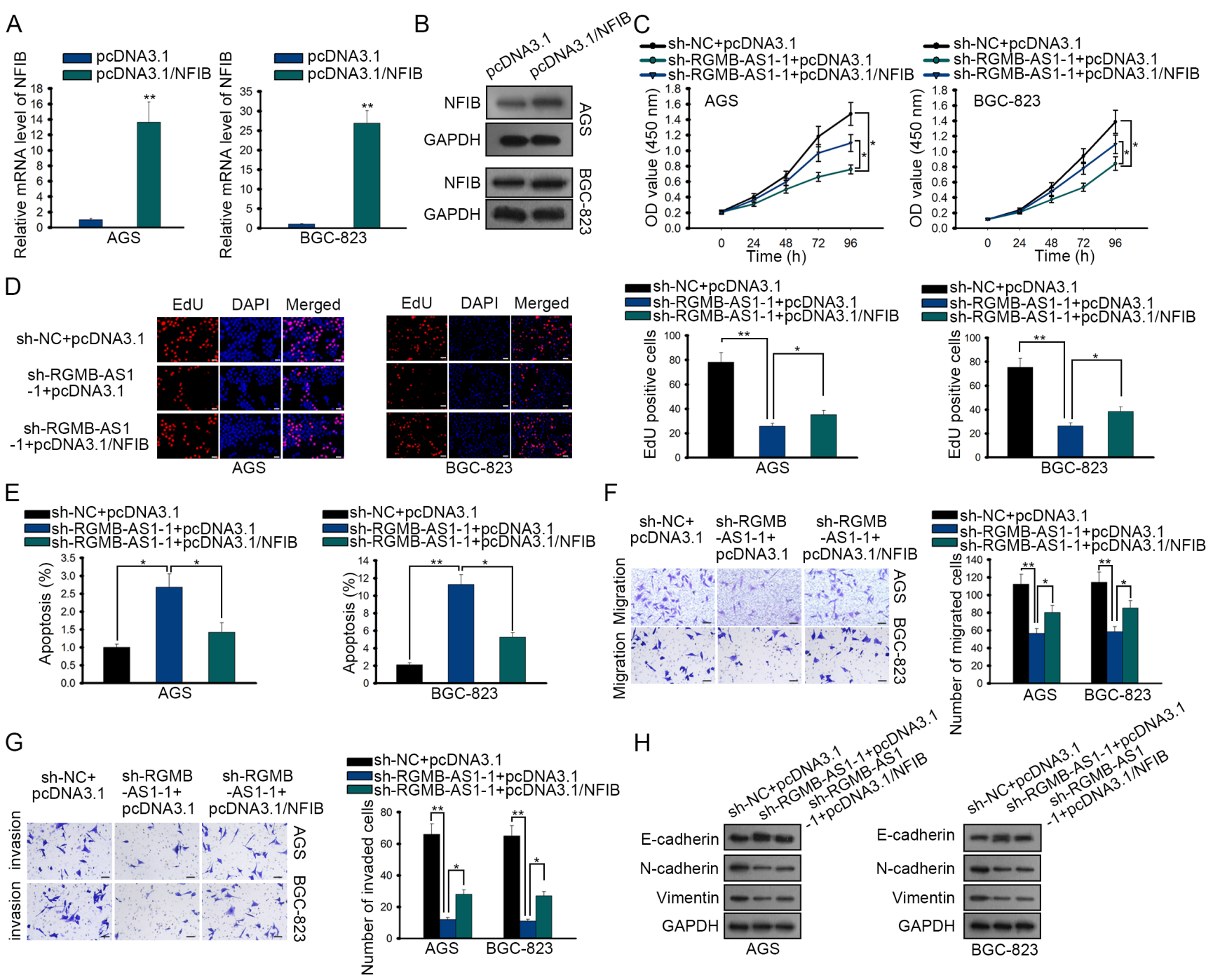

Figure 4. RGMB-AS1 promotes GC by increasing the expression of NFIB. A and B) RT-qPCR and western blot analysis were performed to estimate the overexpression efficiency of pcDNA3.1/NFIB in AGS and BGC-823 cells. C and D) CCK-8 and EdU assays were carried out to detect cell proliferation ability (scale bar $=20 \mu \mathrm{m})$. E) Flow cytometry analysis assay was conducted to evaluate cell apoptosis in GC cells. F and G) Transwell assay was carried out to test cell migration and invasion in GC cells (scale bar $=20 \mu \mathrm{m}$ ). H) Western blot assay was performed to detect the expression of EMT process related proteins. ${ }^{*} \mathrm{p}<0.05,{ }^{* *} \mathrm{p}<0.01$.

anti-IgG (Figure 3F). Additionally, by the use of RT-qPCR, the overexpression efficiency of pcDNA3.1/RGMB-AS1 was measured, showing that the overexpression of RGMB-AS1 evidently increased the expression of RGMB-AS1 in GC cells (Figure 3G). Finally, the luciferase reporter analysis was performed to verify the interaction among RGMB-AS1, miR-22-3p, and NFIB in GC cells. The results presented that the miR-22-3p overexpression declined the expression of NFIB while overexpression of RGMB-AS1 abolished the depletion of NFIB expression caused by the miR-22-3p mimics (Figure $3 \mathrm{H}$ ). All the data indicated that RGMB-AS1 sponges miR-22-3p to regulate NFIB expression in GC.

RGMB-AS1 contributes to GC progression through regulating NFIB. A series of rescue experiments were conducted to detect whether RGMB-AS1 contributed to GC progression through regulating NFIIB expression. After transfection with pcDNA3.1 vectors, the mRNA and protein levels of NFIB were both markedly increased in GC cells (Figures $4 \mathrm{~A}-\mathrm{B}$ ). CCK-8 and EdU assays were implemented to explore cell proliferation in GC cells, exhibiting that the obvious decline of cell proliferation resulted from RGMB-AS1 depletion was partially rescued by NFIB overexpression (Figures 4C-D). As for cell apoptosis, flow cytometry analysis was carried out, showing that the promoting effect caused by suppression of RGMB-AS1 on cell apoptosis was partly abolished by the NFIB overexpression (Figure 4E). To check cell migration, the transwell assay was conducted and demonstrated that the NFIB overexpression partly 
restored the impaired migration or invasion capacity as a result of the RGBM-AS1 knockdown (Figures 4F-G). The increased expression of E-cadherin, the obvious declined expression of $\mathrm{N}$-cadherin and Vimentin resulted from RGMB-AS1 depletion was partially rescued by NFIB overexpression (Figure $4 \mathrm{H}$ ). Collectively, all the data confirmed that RGMB-AS1 contributes to GC progression through regulating NFIB expression.

\section{Discussion}

Previous studies suggested that lncRNAs are engaged in many cancers, such as GC, lung cancer and bladder cancer [11-13]. RGMB-AS1 has been reported to be involved in the progression of cancers. It has been discovered that long non-coding RNA RGMB-AS1 promotes the development and progression of lung adenocarcinoma and non-small cell lung cancer $[9,10]$. Nevertheless, neither the biological function nor the molecular mechanisms of lncRNA RGMB-AS1 in GC have been explored. In this study, it was discovered that the relative expressions of RGMB-AS1 in cells were considerably higher than that in normal gastric cells. Knockdown of RGMB-AS1 reduced cell proliferation, migration, invasion and EMT process, promoted the apoptosis in GC cells. All these data indicated that RGMB-AS1 promoted the progression of GC.

MicroRNA (miRNA), distinguished from other RNAs by its length of 20-24 nucleotides, plays significant roles in the post-transcriptional regulation in gene expression [14, 15]. Recently, it has been discovered that lncRNAs regulate multiple diseases through sponging specific miRNAs [15, 17]. For instance, long non-coding RNA FLVCR1-AS1 functions as an oncogene by sponging miR-155 and targeting c-Myc [18]. LncRNA SNHG5/miR-32 axis modulates GC cell proliferation and migration by targeting KLF4 [19]. LncRNA SNHG16 sponges miR-135a and promotes JAK2/STAT3 pathway in GC [20]. But the interaction of RGMB-AS1 with target miRNAs in GC cells was still obscure. In our current study, we selected miR-22-3p from the potential target miRNAs for RGMB-AS1 on starBase online website. MiR-22-3p has been reported to suppress tumorigenesis in multiple cancers $[21,22]$. In the current study, we further proved the binding ability and the negative interaction between RGMB-AS1 and miR-22-3p.

NFIB has been reported to promote tumorigenesis in human cancers [23-25]. For example, NFIB promotes cell survival by directly suppressing p 21 transcription in TP53-mutated triple-negative breast cancer [23]. NFIB promotes cell growth, aggressiveness, metastasis and EMT of GC through the Akt/Stat3 signaling pathway [24]. The present study confirmed that miR-22-3p could bind to NFIB. Moreover, we found that the downregulation of RGMB-AS1 can decrease NFIB expression while downregulation of miR-22-3p can rescue the reduction of NFIB expression. Finally, rescue assays indicated that overexpression of NFIB partially rescued RGMB-AS1 knockdown-mediated inhibitive effect on GC progression.

To put it in a nutshell, our study proved that RGMB-AS1 accelerated the progression of GC by targeting miR-22-3p/ NFIB axis, indicating the potential of RGMB-AS1 as a new underlying therapeutic target for GC to improve prognosis.

Acknowledgements: We sincerely thank all the involvers for their supports.

\section{References}

[1] BRAY F, FERLAY J, SOERJOMATARAM I, SIEGEL RL, TORRE LA et al. Global cancer statistics 2018: GLOBOCAN estimates of incidence and mortality worldwide for 36 cancers in 185 countries CA Cancer J Clin 2018; 68: 394-424. https://doi.org/10.3322/caac.21492

[2] STRICKLER JH, WU C, BEKAII-SAAB T. Targeting BRAF in metastatic colorectal cancer: Maximizing molecular approaches. Cancer Treat Rev 2017; 60: 109-119. https://doi. org/10.1016/j.ctrv.2017.08.006

[3] LIN MT, SONG HJ, DING XY. Long non-coding RNAs involved in metastasis of gastric cancer. World J Gastroenterol 2018; 24: 3724-3737. https://doi.org/10.3748/wjg.v24. i33.3724

[4] YUSEFI AR, BAGHERI LANKARANI K, BASTANI P, RADINMANESH $M$ et al. Risk Factors for Gastric Cancer: A Systematic Review. Asian Pac J Cancer Prev 2018; 19: $591-$ 603. https://doi.org/10.22034/APJCP.2018.19.3.591

[5] SCHMITT AM, CHANG HY. Long Noncoding RNAs in Cancer Pathways. Cancer Cell 2016; 29: 452-463. https://doi. org/10.1016/j.ccell.2016.03.010

[6] SCHMITZ SU, GROTE P, HERRMANN BG. Mechanisms of long noncoding RNA function in development and disease. Cell Mol Life Sci 2016; 73: 2491-2509. https://doi. org/10.1007/s00018-016-2174-5

[7] GUO X, YANG Z, ZHI Q, WANG D, GUO L et al. Long noncoding RNA OR3A4 promotes metastasis and tumorigenicity in gastric cancer. Oncotarget 2016; 7: 30276-30294. https://doi.org/10.18632/oncotarget.7217

[8] CHEN DL, JU HQ, LU YX, CHEN LZ, ZENG ZL et al. Long non-coding RNA XIST regulates gastric cancer progression by acting as a molecular sponge of miR-101 to modulate EZH2 expression. J Exp Clin Cancer Res 2016; 35: 142. https://doi.org/10.1186/s13046-016-0420-1

[9] LI P, ZHANG G, LI J, YANG R, CHEN S et al. Long Noncoding RNA RGMB-AS1 Indicates a Poor Prognosis and Modulates Cell Proliferation, Migration and Invasion in Lung Adenocarcinoma. PLoS One 2016; 11: e0150790. https://doi. org/10.1371/journal.pone.0150790

[10] LI P, LI J, YANG R, ZHANG F, WANG H et al. Study on expression of lncRNA RGMB-AS1 and repulsive guidance molecule b in non-small cell lung cancer. Diagn Pathol 2015; 10: 63. https://doi.org/10.1186/s13000-015-0297-x

[11] HU J, QIAN Y, PENG L, MA L, QIU T et al. Long Noncoding RNA EGFR-AS1 Promotes Cell Proliferation by Increasing EGFR mRNA Stability in Gastric Cancer. Cell Physiol Biochem 2018; 49: 322-334. https://doi.org/10.1159/000492883 
[12] ZHU Y, DAI B, ZHANG H, SHI G, SHEN Y et al. Long noncoding RNA LOC572558 inhibits bladder cancer cell proliferation and tumor growth by regulating the AKT-MDM2p53 signaling axis. Cancer Lett 2016; 380: 369-374. https:// doi.org/10.1016/j.canlet.2016.04.030

[13] PARK SM, CHOI EY, BAE DH, SOHN HA, KIM SY et al. The LncRNA EPEL Promotes Lung Cancer Cell Proliferation Through E2F Target Activation. Cell Physiol Biochem 2018; 45: 1270-1283. https://doi.org/10.1159/000487460

[14] D'ANGELO B, BENEDETTI E, CIMINI A, GIORDANO A. MicroRNAs: A Puzzling Tool in Cancer Diagnostics and Therapy. Anticancer Res 2016; 36: 5571-5575. https://doi. org/10.21873/anticanres.11142

[15] SRIVASTAVA SK, BHARDWAJ A, LEAVESLEY SJ, GRIZZLE WE, SINGH S et al. MicroRNAs as potential clinical biomarkers: emerging approaches for their detection. Biotech Histochem 2013; 88: 373-387. https://doi.org/10.3109/1 0520295.2012.730153

[16] CUI Y, YI L, ZHAO JZ, JIANG YG. Long Noncoding RNA HOXA11-AS Functions as miRNA Sponge to Promote the Glioma Tumorigenesis Through Targeting miR-140-5p. DNA Cell Biol 2017; 36: 822-828. https://doi.org/10.1089/ dna.2017.3805

[17] LI T, MENG XL, YANG WQ. Long Noncoding RNA PVT1 Acts as a "Sponge" to Inhibit microRNA-152 in Gastric Cancer Cells. Dig Dis Sci 2017; 62: 3021-3028. https://doi. org/10.1007/s10620-017-4508-Z

[18] LIU Y, GUO G, ZHONG Z, SUN L, LIAO L et al. Long noncoding RNA FLVCR1-AS1 sponges miR-155 to promote the tumorigenesis of gastric cancer by targeting c-Myc. Am J Transl Res 2019; 11: 793-805.
[19] ZHAO L, HAN T, LI Y, SUN J, ZHANG S et al. The lncRNA SNHG5/miR-32 axis regulates gastric cancer cell proliferation and migration by targeting KLF4. FASEB J 2017; 31: 893-903. https://doi.org/10.1096/fj.201600994R

[20] WANG X, KAN J, HAN J, ZHANG W, BAI L et al. LncRNA SNHG16 Functions as an Oncogene by Sponging MiR-135a and Promotes JAK2/STAT3 Signal Pathway in Gastric Cancer. J Cancer 2019; 10: 1013-1022. https://doi.org/10.7150/ jca.29527

[21] LV KT, LIU Z, FENG J, ZHAO W, HAO T et al. MiR-22$3 p$ Regulates Cell Proliferation and Inhibits Cell Apoptosis through Targeting the eIF4EBP3 Gene in Human Cervical Squamous Carcinoma Cells. Int J Med Sci 2018; 15: 142-152. https://doi.org/10.7150/ijms.21645

[22] LIU Y, LI H, LIU Y, ZHU Z. MiR-22-3p targeting alphaenolase 1 regulates the proliferation of retinoblastoma cells. Biomed Pharmacother 2018; 105: 805-812.

[23] LIU RZ, VO TM, JAIN S, CHOI WS, GARCIA E et al. NFIB promotes cell survival by directly suppressing p21 transcription in TP53-mutated triple-negative breast cancer. J Pathol 2019; 247: 186-198. https://doi.org/10.1002/path.5182

[24] WU C, ZHU X, LIU W, RUAN T, WAN W et al. NFIB promotes cell growth, aggressiveness, metastasis and EMT of gastric cancer through the Akt/Stat3 signaling pathway. Oncol Rep 2018; 40: 1565-1573. https://doi.org/10.3892/ or.2018.6574

[25] WU Y, ZHANG J, HOU S, CHENG Z, YUAN M. Non-small cell lung cancer: miR-30d suppresses tumor invasion and migration by directly targeting NFIB. Biotechnol Lett 2017; 39: 1827-1834. https://doi.org/10.1007/s10529-017-2428-9 